\title{
UHF RFID split-ring resonator tag antenna inductively coupled feed for metallic object
}

\begin{abstract}
In this paper, a split-ring resonator and an inductively coupled feed technique are designed to improve the RFID tag antennas performance. The presented tag antenna consists of two symmetrical C-shaped resonators with strip line in order to feed the radiating split-ring resonator structures by implementing an inductively coupled feed approach placed on the upper surface of Polytetrafluoroethylene substrate. By selecting a proper length and width of spilt-ring resonator structures, along with desirable coupling distance between two symmetrical C-shaped resonators and spilt-ring resonator structures, the input impedance of tag antenna can be obtained, which leads to attain an excellent conjugate match between antenna and IC chip. The proposed feeding technique and spilt-ring resonator structures offer a better performance of RFID tag among antenna size, reflection coefficient, and gain. The gain of the designed tag antenna is $-2.272 \mathrm{~dB}$ at its operating frequency $(916 \mathrm{MHz})$, the tag antenna is mounted on a square perfect electrical conductor of $200 \mathrm{~mm}$ side length, while the thickness of PEC is $1 \mathrm{~mm}$. The simulation results were verified by the presented method via enhancing the performance of tag antennas for metallic object.
\end{abstract}

Keyword: Antenna gain; Input impedance; Passive tag; Radio frequency identification (RFID); Split-ring resonator (SSR) 